\title{
Einführung in die Diagnostik humanpathogener Pilze - Teil 1: Allgemeiner Teil
}

\author{
Introduction to the Diagnosis of Human Pathogenic Fungi - Part 1: General
}

Autoren

Institut
A. M. Ksoll, B. Sorhage

Abteilung Dermatologie, Venerologie und Allergologie, Bundeswehrzentralkrankenhaus Koblenz
Bibliografie

Dol http://dx.doi.org/ $10.1055 / \mathrm{s}-0030-1256758$ Akt Dermatol 2011; 37: 347-352 @ Georg Thieme Verlag KG Stuttgart · New York ISSN 0340-2541

Korrespondenzadresse Anna Maria Ksoll

Biomed. Fachanalytikerin und Fachlehrerin (LGW-Univ.)

Dr. med. Bernhard Sorhage Abteilung Dermatologie, Venerologie und Allergologie Bundeswehrzentralkrankenhaus Koblenz

Akademisches Lehrkrankenhaus der Johannes Gutenberg Universität Mainz Rübenacher Straße 170 56072 Koblenz

\section{Zusammenfassung $\nabla$}

Die Pilze (Fungi) sind chlorophyllose, meist myzelbildende Thalophyten und in der Natur ubiquitär verbreitet. Nur relativ wenige der sehr zahlreichen, auf ungefähr 200000 und mehr geschätzten Pilzarten sind humanpathogen und können Mykosen der Haut, der Schleimhäute und der inneren Organe hervorrufen. Die Identifizierung der Pilze erfolgt in zwei getrennten Schritten, und zwar mithilfe eines mikroskopischen Direktpräparates, ein sogenanntes Nativpräparat, und einer kulturellen Untersuchung. In der parasitären Phase werden - im Gegensatz zur saprophytären (conidialen) Phase - keine charakteristischen Formelemente gebildet, und die mikroskopische Untersuchung von Nativpräparaten beschränkt sich ausschließlich auf den Nachweis

\section{Kurze Einführung in die Medizinische Mykologie}

Pilze sind chlorophyllose, eukaryontische, wenig differenzierte, zumeist myzelbildende Thalophyten mit charakteristischen Zellwänden, deren Matrix u. a. aus Chitin besteht.

Durch das Fehlen von Chromatophoren sind sie nicht zur eigenen Fotosynthese befähigt, sondern als Saprophyten oder Parasiten auf Kohlenstoffverbindungen anderer Organismen angewiesen. In der biologischen Klassifikation bilden sie neben Tieren und Pflanzen ein eigenständiges Reich und stellen mit ca. 200000 Arten eine sehr große und ungewöhnlich formenreiche Gruppe dar.

Nur relativ wenige der sehr zahlreichen, in unserer Umwelt vorkommenden Pilzarten können als Krankheitserreger beim Menschen in Erscheinung treten und Mykosen der Haut und ihrer Anhangsgebilde (Haare und Nägel), der Schleimhäute und der inneren Organe hervorrufen. Je nach von Pilzfäden und Sporen. Nativpräparat und Kultur stimmen nicht immer überein. In der Pilzdiagnostik sind infolgedessen stets beide Methoden anzuwenden. Das Nativpräparat sagt nichts über die Pilzart aus. Eine Differenzierung ist nur mithilfe einer kulturellen Untersuchung möglich. Die Anzüchtung erfolgt auf festen (Schrägagar-Reagenzglas- und Petrischalenkulturen) oder flüssigen Nährböden mit organischem Kohlenstoff- und Stickstoffgehalt. Die in den Kulturen wachsenden Pilze werden entsprechend dem D-H-S-System in drei Gruppen eingeteilt. Man unterscheidet Dermatophyten (D), Hefen (H) und Schimmelpilze (S). Die Dermatophyten zeichnen sich immer - ebenso wie die Schimmelpilze - durch Bildung von Luftmyzel aus und sind in der Regel nach 1-3 Wochen Bebrütung bei $22-28^{\circ} \mathrm{C}$ zu identifizieren.

Pilzart können entweder disponierende Faktoren vorhanden sein (z.B. bei opportunistischen mykotischen Infektionen) oder auch fehlen (z. B. bei Dermatophytosen) [1].

Der Lebenszyklus der Pilze läuft in zwei verschiedenen Phasen ab. Man unterscheidet eine imperfekte, unvollkommene, vegetative Form (Anamorph) und eine perfekte, vollkommene Form (Telemorph) mit sexueller Fortpflanzung unter Bildung typischer Fruchtkörper mit Geschlechtssporen, die für die Einordnung der Pilze in das Ordnungssystem ausschlaggebend sind.

Die beiden Vermehrungsphasen haben unterschiedliche Aufgaben zu erfüllen. Während die perfekte Form mit ihren sexuellen Fruchtformen bzw. Hauptfruchtformen, wie z.B. den Ascosporen, Zygosporen und Basidiosporen, auch der Evolution dient, sorgt die imperfekte Form mit ihren sehr zahlreichen, charakteristischen asexuellen Fruchtformen, den sogenannten Nebenfruchtformen, wie z.B. den Makro- und Mikroconidien, 
Blastosporen, Chlamydosporen, Arthrosporen, Sporangiosporen usw., vor allem für die Erhaltung, Vermehrung und Verbreitung der Art.

Pilze, die sich ungeschlechtlich vermehren oder deren Sexualformen nicht bekannt sind, werden in ein künstliches System der „Fungi imperfecti“ eingeordnet und als Deuteromyzeten bezeichnet.

Aufgrund von Merkmalen ihrer sexuellen Vermehrung werden die Pilze (nach Müller und Loeffler) in folgende Abteilungen und Klassen eingeteilt:

1. Zygomycota (jochpilzartige)

a) Zygomycetes

b) Trichomycetes

2. Ascomycota (schlauchpilzartige)

a) Endomycetes

b) Ascomycetes

3. Basidiomycota (ständerpilzartige)

a) Ustomycetes

b) Basidiomycetes

Eine andere Einteilungsmöglichkeit der Pilze ist die nach der Morphologie, wobei Hyphenpilze, Sprosspilze (Hefen) und dimorphe Pilze unterschieden werden.

In der Medizinischen Mykologie hat sich jedoch vor allem die einfache Einteilung der Mikropilze in Dermatophyten, Hefen und Schimmelpilze bewährt.

Die Hyphenpilze (hierzu gehören die Dermatophyten und Schimmelpilze) bilden fadenförmige Zellen, die als Hyphen und deren Geflecht als Myzel bezeichnet werden. Das submers wachsende, sogenannte Substralmyzel hat die Aufgabe, Nährstoffe aufzunehmen, während das Luft- bzw. Oberflächenmyzel, das sich zu einer annähernd runden Kolonie entfaltet, mit seinen meist sehr zahlreichen, charakteristischen, der Fortpflanzung dienenden Konidiosporen die Vermehrung der Art gewährleistet.

Die Dermatophyten und die Schimmelpilze unterscheiden sich von den Sprosspilzen, die von cremeartiger, schleimiger oder pastöser Konsistenz sind, insbesondere durch die Bildung von Luftmyzel. Die Sprosspilze vermehren sich vegetativ durch Sprossung bzw. Knospung. Die Sprossung kann entweder unipolar, bipolar oder an vielen Stellen der „Mutterzelle“ (multilokulär gleichzeitig) erfolgen.

Charakteristische Mikrostrukturen der Sprosspilze sind

1. die Sprosszellen, die sogenannten Blastosporen,

2. die Chlamydosporen (Dauersporen bzw. Mantelsporen), die mit Reservestoffen angereichert sind und die Erhaltung der Vitalität einer Kultur über sogenannte „Dormanz-Perioden“ sichern,

3. die Arthrosporen, die durch Fragmentation der Hyphen entstehen, sowie

4. Pseudohyphen, die aus langgestreckten, aneinander hängenden Blastosporen bestehen und deren Geflecht als

Pseudomyzel bezeichnet wird, und

5. echtes Myzel, das jedoch nur ausnahmsweise vorhanden ist. Die dimorphen Pilze wachsen bei $22-28^{\circ} \mathrm{C}$ in ihrer saprophytären, myzelbildenden Phase und vermögen bei $37^{\circ} \mathrm{C}$ in die parasitäre Hefephase überzugehen, wobei sich die Morphologie entsprechend ändert und Sprosszellen an Stelle der ursprünglichen, zahlreichen, charakteristischen Myzelstrukturen gebildet werden. Der Dimorphismus ist ein charakteristisches Merkmal einiger Pilzerreger systemischer Mykosen, wie z.B. der Sporothrixund Histoplasma-Mykose sowie der nordamerikanischen Blastomykose.

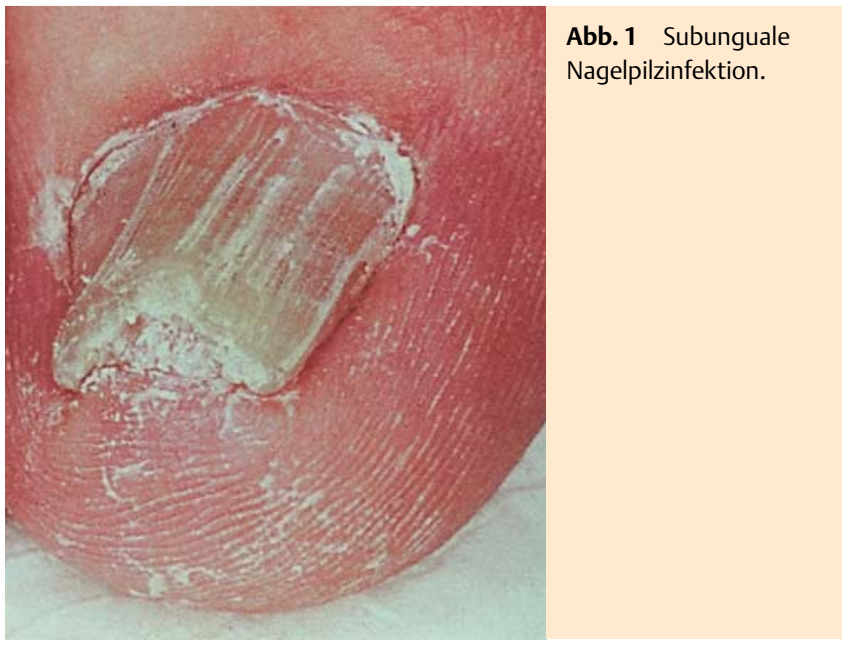

Die Identifizierung der Dermatophyten und Schimmelpilze erfolgt aufgrund einer Vielzahl makro- und mikromorphologischer Merkmale:

Von diagnostischer Bedeutung sind Koloniengröße, -form (Oberflächenstruktur, Faltenbildung, Randbeschaffenheit) und -farbe (Ober- und Unterseite). Entscheidend für die Bestimmung der Gattungs- und Artzugehörigkeit dieser Pilze sind jedoch vor allem die asexuellen Fruchtformen bzw. Nebenfruchtformen sowie eine Reihe anderer, in der imperfekten Form gebildeter charakteristischer Mikrostrukturen.

Die Identifizierung der Sprosspilze erfolgt durch morphologische Beurteilung der charakteristischen Mikrostrukturen auf Reisagar sowie durch Bestimmung biochemischer Eigenschaften (Fermentations- und Assimilationsleistungen).

\section{Gewinnung des Untersuchungsmaterials}

Die Erfolgsquote bei der Beurteilung von Nativpräparaten und der Identifizierung von Pilzen steht in direktem Zusammenhang zur richtigen Materialentnahme. Die Gewinnung des Untersuchungsmaterials, die durch eine sehr gründliche mechanische und chemische Oberflächenreinigung mithilfe von sterilen Mulltupfern und 70\%igem Ethyl- oder 50\%igem Isopropylalkohol eingeleitet wird (sogenannte „Pilzherd-Toilette“ nach Rieth) ist jeweils von dem Untersuchungsort (Haut, Haare oder Nägel) des mykoseverdächtigen Krankheitsherdes abhängig [2].

\section{Hautschuppen}

Die pilzverdächtigen Hautherde werden nach Entfernung der oberflächlichen Auflagerungen (Krustenbildung und groben Schuppen) mit einem Alkoholtupfer weitgehend gereinigt. Anschließend werden ca. 40-50 Hautschüppchen (etwa $1 \mathrm{~mm}$ kleine Teilchen) mit einem stumpfen Skalpell oder scharfen Löffel aus der Peripherie des Herdes entnommen. In dieser Zone lebhaften Pilzwachstums findet man junges Myzel, das sich im Präparat besonders gut darstellen lässt [3].

\section{Nagelmaterial}

Man unterscheidet die subunguale und oberflächliche Nagelpilzinfektion. Beim subungualen Typ ( $\bullet$ Abb. 1) werden Nagelspäne nach Entfernung der leicht ablösbaren, bröckeligen sowie grob veränderten Nagelanteile und anschließender sorgfältiger Reinigung aus der Tiefe des Nagelbetts (mit Brocque'scher Kürette, 


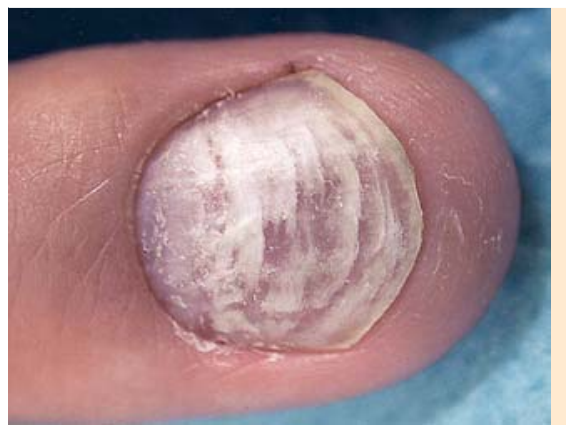

Abb. 2 Oberflächliche Nagelpilzinfektion.

stumpfem Skalpell oder scharfem Löffel) herausgelöst. Beim oberflächlichen Typ ( $\bullet$ Abb. 2) sind Nagelspäne von der Nageloberfläche zu gewinnen.

Die Entnahme des Untersuchungsmaterials (mindestens 20 kleine Nagelteilchen) sollte an Stellen erfolgen, wo krankes Gewebe unmittelbar an gesundes Gewebe anschließt.

\section{Haare}

Bei Verdacht auf Pilzbefall von Kopf- oder Körperhaaren werden, nach sorgfältiger Reinigung und Entfernung der um den mykoseverdächtigen Herd herum vorkommenden Krusten und groben Schuppen, etwa 10-20 Haarstümpfe einzeln und sehr vorsichtig mittels einer Epilationspinzette vom Rande der Herde ausgezupft; Haare nicht mit der Schere herausschneiden.

Beim Abrisspräparat, der häufigsten Nachweismethode einer Tinea versicolor, wird das Nativmaterial durch Aufkleben eines Tesafilm-Klebestreifens auf einen mykoseverdächtigen Herd gewonnen. Der Klebestreifen wird anschließend wieder abgezogen, auf einen Objektträger aufgebracht und das Präparat mit oder ohne Färbung bei 400-facher Vergrößerung mikroskopiert.

Malassezia furfur bildet überwiegend nestförmig gelagerte Sporen sowie relativ kurze, wurstförmig gekrümmte Pilzfäden und ist aus dem Abrisspräparat sicher zu identifizieren.

Von der Mundschleimhaut, der Vagina und vom Perianalbereich wird das Untersuchungsmaterial mit einem sterilen Abstrichtupfer oder einer sterilen Impföse gewonnen. Die Mundhöhle sollte vorher sorgfältig mit $0,9 \%$ iger $\mathrm{NaCl}$-Lösung ausgespült werden. Das Probenmaterial wird anschließend in einem handelsüblichen Versandröhrchen mit Transportmedium zur mykologischen Untersuchung gegeben.

Beim Abklatsch-Verfahren, der häufigsten Nachweismethode einer Candida-Infektion des männlichen Genitals, wird ein gebrauchsfertiger Pilznährboden mehrmals vorsichtig gegen die Glans penis und/oder das Präputium gedrückt. In ähnlicher Weise wird bei Abklatsch-Kulturen von der Zunge verfahren.

Stuhlproben werden unmittelbar nach der Ausscheidung mit einem sterilen Abnahmelöffelchen an mehreren Stellen der Stuhlportion entnommen und in einem dafür bestimmten sterilen Gefäß zur mykologischen Untersuchung gegeben.

\section{Direkte mikroskopische Untersuchung}

$\nabla$

Für die mikroskopische Direktuntersuchung muss das Nativmaterial entsprechend vorbehandelt werden. Die Aufbereitung des Materials erfolgt mit 10\%-, 15\%- oder 20\%iger Kalilauge, die

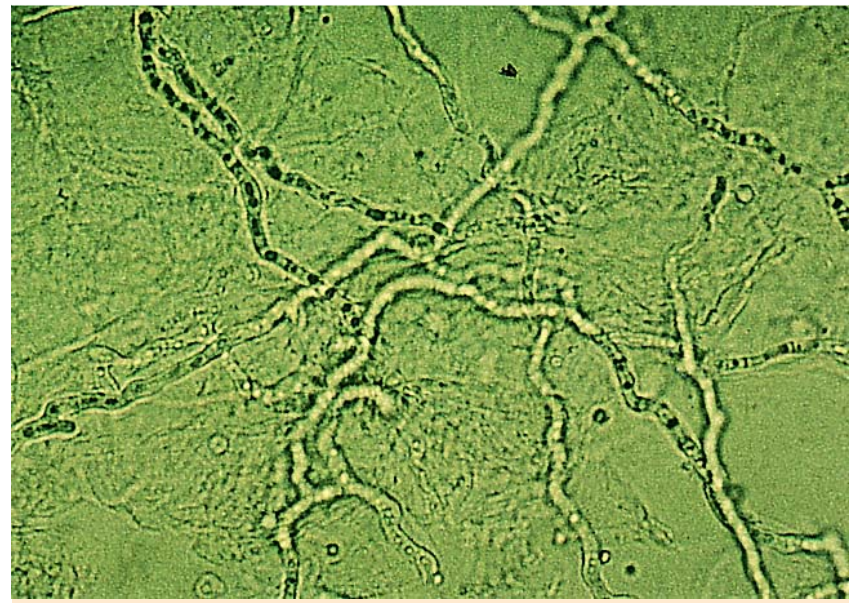

Abb. 3 Hyphen im Nativpräparat (stark vergrößert).

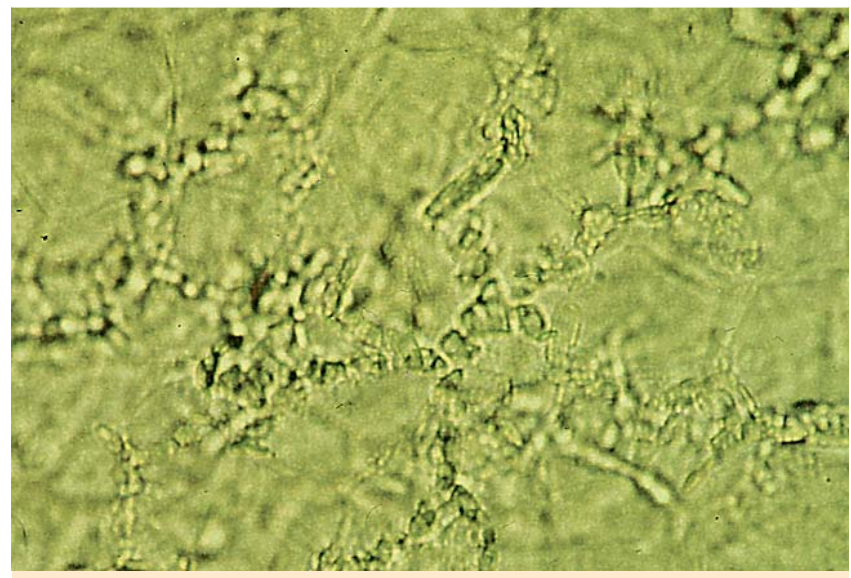

Abb. 4 Mosaikfungi im Nativpräparat (stark vergrößert).

eine weitgehende Mazeration der keratinhaltigen Proben sowie eine Aufhellung der Pilzelemente bewirkt. Nach einer Einwirkungszeit von etwa 30-60 Minuten in der sogenannten ,feuchten Kammer" sind die Pilzfäden und Sporen in den Hautschuppen, Nagelspänen und Haaren infolge Auflösung der Hornsubstanz viel besser zu erkennen.

Zur raschen Orientierung in der Praxis empfiehlt es sich, anstelle der Kalilauge eine 10\%ige Tetraethylammoniumhydroxyd-(TEAH-)Lösung zu verwenden. Durch diese Lösung wird der Mazerationsvorgang wesentlich beschleunigt und die Pilzelemente können in der Regel schon nach wenigen Minuten im Nativpräparat nachgewiesen werden.

Die Nativpräparate werden zunächst bei 10-facher Objektivvergrößerung durchmustert. Findet man eine suspekte Stelle, so ist anschließend eine Untersuchung bei 40 -facher Objektivvergrößerung angezeigt.

In der parasitären Phase ist das Erscheinungsbild - im Gegensatz zur saprophytären (conidialen) Phase - extrem vereinfacht und die mikroskopische Untersuchung von Nativpräparaten beschränkt sich ausschließlich auf den Nachweis von Pilzfäden ( Abb. 3) und Sporen.

In den Nativpräparaten findet man relativ häufig als chemisches Reaktionsprodukt infolge $\mathrm{KOH}$ - oder TEAH-Einwirkung sogenannte „Mosaikfungi“ ( $\bullet$ Abb.4). Diese hyphenähnlichen Artefakte können von „Ungeübten“ gelegentlich mit echtem Myzel verwechselt werden. Im Gegensatz zum Myzel weisen diese 
Artefakte jedoch keine Septierung und keine echte Verzweigung auf [4].

Das Nativpräparat sagt nichts über die Pilzart aus. Gewisse Anhaltspunkte erlaubt lediglich das Wachstum auf Haaren, das endothrich (= in den inneren Schichten des Haares) oder ektothrich (= auf den äußeren Schichten des Haares) sein kann. Eine genaue Differenzierung ist nur mithilfe einer kulturellen Untersuchung möglich. Die Anzüchtung erfolgt auf festen (Schrägagar-Reagenzglas- und Petrischalenkulturen) oder flüssigen Nährböden mit organischem Kohlenstoff- und Stickstoffgehalt.

\section{Anlegen einer Makrokultur}

$\nabla$

Das Nativmaterial wird mittels steriler Öse oder sterilem Impfhaken Stück für Stück einzeln auf die Agarplatte in einer Petrischale verteilt und leicht eingedrückt, damit es guten Kontakt zum Nährboden hat.

Es sind etwa 20 - 30 Hautschuppen oder 10-20 kleine Nagelteilchen zu inokulieren und mindestens 10 Haarstümpfe sorgfältig in den Agar einzubetten.

Auf Schrägagar wird das Nativmaterial an mehreren Inokulationsstellen im Winkel zwischen Agar und Glaswand sowie in der Agarmitte verimpft.

Sekrete werden zickzackförmig ausgestrichen.

Es empfiehlt sich von jedem Patienten vier Schrägagar-Röhrchen oder zwei Plattenkulturen (mit und ohne Zusatz von Cycloheximid) anzulegen.

Wird jeweils nur ein Röhrchen oder eine Petrischale beimpft, so besteht die Gefahr falsch-negativer Untersuchungsergebnisse. Um eine Kontamination durch Luftkeime zu vermeiden, werden die beimpften Kulturplatten mit dem Deckel nach oben bebrütet.

\section{Zur Beachtung: Pilzfressende Milben}

Im Hausstaub befinden sich häufig verschiedene Arten von Milben, z.B. der Gattung Tyrophagus und Tarsonemus, die in die Petrischalenkulturen einwandern, die „Pilzrasen abweiden“, Bakterien sowie andere unerwünschte Begleitkeime (Schimmel- und Hefepilze) verimpfen und deshalb in Pilzsammlungen (Mykotheken) ständig bekämpft werden müssen.

\section{Techniken zum Studium der Mikromorphologie $\nabla$}

Für die mikroskopische Differenzierung von Dermatophyten und Schimmelpilzen sind am besten folgende Techniken geeignet:

Objektträger-Deckglas-Kultur (nach Rivalier und Riddell) Aus einem Fertignährboden werden Agarwürfel von je ca. $1 \times 1 \mathrm{~cm}$ ausgeschnitten, auf sterile Objektträger gelegt, an den Schnittflächen beimpft, anschließend mit sterilen Deckgläschen bedeckt und in einer feuchten Kammer bei Raumtemperatur bebrütet.

\section{Petrischalen-Mikrokultur (nach Ito und Refai)}

Mehrere ca. $2 \mathrm{~cm}$ lange und 0,5 cm breite Agarblöckchen werden aus einem Fertignährboden herausgeschnitten und verworfen. Anschließend wird an je drei Seiten beimpft, ein steriles Deckgläschen über den beimpften Bereich so gelegt, dass noch ein Luftloch bestehen bleibt, und bei Raumtemperatur bebrütet.

\section{Tesafilm-Methode (nach Rebell und Taplin)}

Ein Tesafilmklebestreifen wird auf die Oberfläche eines Kolonienzentrums leicht aufgedrückt, wieder abgezogen und anschließend auf einem Objektträger fixiert. Die Präparate können mit einer Farblösung, wie z. B. Methylenblau- oder Lactophenolblaulösung, angefärbt werden.

Die mikromorphologische Beurteilung von Sprosspilzen erfolgt am besten auf der Reisagarplatte (nach Taschdjian und Rieth), die aus Reisdekokt und Agar-Agar hergestellt wird. Nach sehr dünner Strichimpfung werden sterile Deckgläser aufgelegt und die geschlossenen Petrischalen 48 - 72 Stunden bei Raumtemperatur bebrütet.

\section{Wood-Licht}

Bei Verdacht auf Pilzbefall von Kopf- oder Körperhaaren wird relativ häufig die sogenannte „Wood-Lampe“ als diagnostisches Hilfsmittel angewendet. Die Lampe besteht aus einer Quecksilberhochdrucklampe und einem Nickeloxidfilter, durch das nur die Ultraviolett-A-Strahlen mit einem Wellenbereich um $365 \mathrm{~nm}$ hindurchgehen. Diese langwelligen Ultraviolett-A-Strahlen führen im abgedunkeltem Raum bei bestimmten oberflächlichen Pilzinfektionen zu fluoreszierendem Aufleuchten.

Bei Microsporum (M.) audouinii, M. canis, M. distortum, M. equinum und $M$. ferrugineum zeigen die pilzbefallenen Haare eine grüne und beim Favus (Trichophyton schoenleinii) eine graugelbe bis grauweiße Fluoreszenz-Färbung. Bei Trichophyton schoenleinii kann zusätzlich ein sogenannter „Hohlraumeffekt“ beobachtet werden, da im endothrich befallenen Haarschaft das Myzel stellenweise dunkel erscheint [5].

Bei Mikrosporien, die von Katzen übertragen werden, wird das Fluoreszenzphänomen vermisst. Der Wood-Licht-Inspektion sind deshalb immer mikroskopische und kulturelle Untersuchungen anzuschließen [6].

\section{Die gebräuchlichsten Nährböden in der Pilzdiagnostik $\nabla$}

\section{Kimmig-Agar}

Rp. (g/Liter)

Glukose 10,0; Pepton 5,0; Standard II Nährbouillon „Merck“ 15,0; Agar 20,0; zusätzlich Glyzerin $5 \mathrm{ml}$.

$\mathrm{pH} 6,5$

Der Kimmig-Agar ist ein mykologischer Universalnährboden, der ein sehr gutes Wachstum aller Dermatophyten, Hefen (ausgenommen der Pityrosporumarten) und Schimmelpilze ermöglicht. Durch den geringen Glukosegehalt wird die Fruchtifikation der meisten humanpathogenen Pilze stimuliert. Die Entwicklung von sterilem, watteartigem Myzel wird bei Verwendung dieses Agars weitesgehend verhindert [7].

\section{Sabouraud-Glukose-Agar}

Rp. (g/Liter)

Glukose 20,0-40,0; Pepton 10,0; Agar 20,0.

$\mathrm{pH} 5,6$

Der Sabouraud-Glukose-Agar enthält bis zu 4\% Glukose und wird ebenfalls sehr häufig für die Anzüchtung, Isolierung und Identifizierung der pathogenen Pilze verwendet. Dieser Universalnährboden fördert aufgrund seines relativ hohen Kohlenhydratanteils die charakteristische Pigmentbildung sowie das Wachstum der Pilze. 


\section{Malzextrakt-Agar}

Rp. (g/Liter)

Maltose 12,75; Malzextrakt 15,00; Glukose 2,75; Glyzerin 2,35; K2HPO4 1,00; NH4Cl 1,00; Pepton 0,78.

pH 6,8-7,0

Der Malzextrakt-Agar verhindert das Pleomorphwerden der Pilzkulturen und eignet sich infolgefolgedessen vor allem für die Anlegung einer Mykothek. Dieser Nährboden stimuliert auch die Makroconidienbildung bei Trichophyton tonsurans.

\section{Selektivagar für pathogene Pilze (Mycosel)}

Rp. (g/Liter)

Glukose 10,0; Pepton 10,0; Agar 15,5-20,0; Cycloheximid 0,4; Chloramphenicol 0,05.

pH 6,9

Der Selektivagar für pathogene Pilze hemmt das Wachstum zahlreicher Begleitkeime (Schimmel- und Hefepilze sowie Bakterien) und sollte bei Verdacht auf starke Verunreinigung des Untersuchungsmaterials parallel zu einem hemmstofffreien Nährboden (z. B. Kimmig- oder Sabouraud-Glukose-Agar) mitbeimpft werden.

Cave! Durch Cycloheximid in den Selektivnährböden kann auch das Wachstum einer Reihe fakultativ-pathogener Pilze (wie z.B. Candida [C.] glabrata, C. krusei, C. parapsilosis, C. tropicalis, Cryptococcus neoformans, Trichosporon cutaneum und Aspergillus fumigatus) gehemmt werden.

\section{Dermatophyten-Selektivagar nach TAPLIN}

Rp. (g/Liter)

Pepton aus Sojamehl; Glukose 10,0; Cycloheximid 0,5; Gentamycinsulfat 0,1; Chlortetracyclin 0,1; Phenolrot 0,2; Agar 17,0.

$\mathrm{pH} 5,5$

Der Dermatophyten-Selektivagar nach Taplin enthält einen Indikator-Farbstoff, der durch intensive Rotfärbung des Agars auf die Anwesenheit eines Dermatophyten hinweist. Dieser SelektivNährboden ist jedoch nicht absolut dermatophytenspezifisch. Einige keratinophile Schimmelpilze, wie z.B. Scopulariopsisund Chrysosporium-Arten, können ebenfalls einen Farbumschlag des Indikators bewirken.

\section{Kartoffel-Glukose-Agar nach Lodder \\ Rp. (g/Liter) \\ Kartoffelstückchen 20,0; Glukose 10,0; Agar 15,0. \\ pH 5,6}

Kartoffelstückchen in Aqua dest. weich kochen, Glukose und Agar zugeben und auf 1 Liter auffüllen.

Der Kartoffel-Glukose-Agar stimuliert die Pigmentinduktion bei Trichophyton rubrum und wird außerdem auch relativ häufig als Universalmedium zur Züchtung und Differenzierung pathogener Pilze verwendet.

\section{Glukose-Pepton-Agar nach Emmons}

Rp. (g/Liter)

Glukose 20,0; Pepton 10,0; Agar 20,0.

$\mathrm{pH} 6,8-7,0$

Der Glukose-Pepton-Agar nach Emmons ist ein universeller Nährboden zur Anzüchtung pathogener Pilze aus bakteriell verunreinigtem Untersuchungsmaterial.
Maismehlagar

Rp. (g/Liter)

Maismehl 40; Agar 20.

Maismehl in Aqua dest. 1 Std. kochen, filtrieren, Agar zugeben und auf 1 Liter auffüllen.

Der Maismehlagar stimuliert die Conidienbildung bei den Dermatophyten. Er ist auch vorzüglich zur Chlamydosporenbildung von Candida albicans geeignet - dann vor dem Autoklavieren $10 \mathrm{~g}$ Tween 80 zugeben [3].

\section{Czapek-Dox-Agar}

Rp. (g/Liter)

Saccharose 30,0; NaNO3 3,00; K2HP04 1,00; MgS04 7 H20 0,50; $\mathrm{KCl}$ 0,50; FeS04 7 H20 0,01; Agar 15,00.

$\mathrm{pH} 7,3$

Der Czapek-Dox-Agar dient vor allem der Anzüchtung, Isolierung und Identifizierung von Aspergillus- und Penicillium-Arten.

\section{Reis-Agar}

Rp. (g/Liter)

Brühreis (keinen Milchreis verwenden) 20,0; Agar 20,0

Der Reis-Agar stellt ein Minimalmedium zur Differenzierung von Sprosspilzen dar. Der geringe Nährstoffgehalt stimuliert zusammen mit sauerstoffarmen Kulturbedingungen die Bildung charakteristischer Mikrostrukturen, vor allem die Chlamydosporenbildung von Candida albicans.

Die Agar-Nährmedien werden mit $2 \mathrm{~N} \mathrm{NaOH}$ oder $2 \mathrm{~N} \mathrm{HCl}$ auf den gewünschten $\mathrm{pH}$ eingestellt und anschließend 15 Minuten bei $120^{\circ} \mathrm{C}$ autoklaviert.

Um das Bakterienwachstum weitgehend zu verhindern, werden den auf $60^{\circ} \mathrm{C}$ abgekühlten Nährmedien antibakterielle Antibiotika (Penicillin + Streptomycin; Chloramphenicol) zugesetzt.

\section{D-H-S-System (nach Rieth)}

\section{$\nabla$}

In der Praxis hat es sich bewährt, die in den Kulturen wachsenden Pilze makroskopisch grob orientierend in drei Gruppen einzuteilen. Man unterscheidet

1. Dermatophyten (D),

2. Hefen $(\mathrm{H})$ und

3. Schimmelpilze (S).

Die Dermatophyten sind keratinophile Pilze und verursachen nur Mykosen der Haut, der Haare und der Nägel, die als Dermatophytosen bezeichnet werden.

Die Kulturen zeichnen sich immer durch Bildung von Luftmyzel aus und sind in der Regel nach 1-3 Wochen Bebrütung bei 22$28^{\circ} \mathrm{C}$ zu identifizieren.

Die Kolonien sind zumeist weißlich-cremefarben, manchmal auch gelb, gelb-braun, orange-braun, rot oder braun pigmentiert, mit samtiger, wildlederähnlicher, flaumiger oder puderig-körniger bis gipsiger, oftmals radiär oder zerebri-/krateriform gefurchter Oberfläche.

Hefemykosen der Haut, der Schleimhäute und der inneren Organe, die sogenannten Levurosen, werden vor allem durch die imperfekten Hefen der Gattung Candida, Cryptococcus, Malassezia (Pityrosporum) und Trichosporon hervorgerufen. Eine Ausnahmestellung nimmt hierbei Malassezia furfur, der Erreger der Pityriasis versicolor, einer oberflächlichen, jedoch rezidivfreudigen, auch als „Kleienflechte“ bezeichneten Dermatomykose ein [8]. 
Die beimpften Kulturplatten werden im Brutschrank bei $37^{\circ} \mathrm{C}$ und parallel dazu bei $22-28^{\circ} \mathrm{C}$ bis zu 5 Tage bebrütet.

Die Kulturen sind von weichpastöser oder schleimiger Konsistenz, mit glatter oder leicht gekräuselter Oberfläche. Stark gefaltete und zerklüftete Kolonien werden nur von Hefen der Gattung Trichosporon gebildet.

Bei Bestehen gravierender disponierender Faktoren, z. B. starker Schwächung des Immunsystems, können auch bestimmte, normalerweise saprophytär lebende Schimmelpilze als fakultativpathogene Krankheitserreger beim Menschen in Erscheinung treten und Schimmelpilz-Mykosen der Haut, der Schleimhäute und vor allem der inneren Organe verursachen.

Die Schimmelpilze bilden ebenso wie die Dermatophyten immer Luftmyzel und werden bis zu 3 Wochen bebrütet.

Die Kolonien sind blau, grün, blau-grün, gelblich, rötlich, bräunlich oder braun-schwarz bis ruß-schwarz pigmentiert, mit flockiger, pelziger oder granulös-staubiger, meist unregelmäßig gefurchter, manchmal zentral gewulsteter Oberfläche.

Die meisten apathogenen, in unserer Umwelt ubiquitär vorkommenden Schimmelpilze wachsen optimal bei Raumtemperatur. Die pathogenen Aspergillus- und Mucorales-Spezies, die Erreger invasiver, opportunistischer Systemmykosen vermehren sich dagegen besser bei $37^{\circ} \mathrm{C}$. Diese Schimmelpilze zeichnen sich durch ein sehr schnelles Wachstum aus und sind in der Regel schon innerhalb von wenigen Tagen zu identifizieren.

\section{Abstract}

\section{Introduction to the Diagnosis of Human Pathogenic Fungi - Part 1: General \\ $\nabla$}

Fungi are thallophytes that lack chlorophyll and generally form mycelia. They are abundant in nature. Only a few of the estimated 200000 or more fungus species are pathogenic for humans and cause mycoses of the skin, mucous membranes, and internal organs.

Fungi are identified in two separate steps. The first is microscopic observation, and the second is culture.

Characteristic microstructures do not develop during the parasitic phase, unlike during the saprotrophic (conidial) phase. The microscopic observation of specimens is limited to the detection of hyphae and spores.

Microscopic observations and cultures do not always correspond. For this reason, both methods must always be applied.

Microscopic observation cannot be used to determine the type of fungus. This is only possible by means of culture. Fungi are cultivated on a solid (agar slant and Petri dish) or liquid culture medium with an organic carbon and nitrogen source.

Fungi growing on cultures are divided into three groups according to the DYM system. DYM stands for dermatophytes (D), yeasts (Y) and moulds (M).

Dermatophytes - like moulds - always form mycelium and can, as a rule, be identified after an incubation period of one to three weeks at $22-28^{\circ} \mathrm{C}$.

\section{Literatur}

1 Qadripur S-A. Pilze und Pilzerkrankungen. Stuttgart: Thieme; 1981

2 Ksoll A-M, Sorhage B. Dermatophyten-Diagnostik. Wehrmedizinische Monatsschrift 2007; 8: 210-218

3 Seeliger HPR, Heymer T. Diagnostik pathogener Pilze des Menschen und seiner Umwelt. Stuttgart: Thieme; 1981

4 Nolting S, Fegeler K. Medizinische Mykologie. 3. Aufl. Heidelberg: Springer; 1987

5 Kozlowska EA, Nuber D. Leitfaden der praktischen Mykologie. Berlin: Blackwell; 1996

6 Heber $W$, Hauss $H$. Mykologische Techniken in der ärztlichen Praxis. München: Schwarzeck; 1983

7 Tietz H-J, Ulbricht $\mathrm{H}$. Humanpathogene Pilze der Haut und Schleimhäute. Hannover: Schlütersche; 1999

8 Ksoll A-M, Sorhage B. Identifizierung von Dermatophyten. MTA Dialog 2005; 9: 654-656 